\section{CANCER}

\section{Enhancing EGFR targeting}

The epidermal growth factor receptor (EGFR) is altered in $50 \%$ of glioblastomas (GBMs), but EGFR inhibitors have yielded disappointing results in clinical assays. A new study may allow improved targeting of EGFR-dependent GBMs by identifying a key regulator of EGFR in GBMs (J. Clin. Invest. http://dx.doi.org/ 10.1172/JCI63623).

Natividad Pozo et al. examined the effect of DYRK1A, a kinase important in central nervous system development, on EGFR in established GBM cell lines. They found that silencing of DYRK1A led to a reduction in the amounts of EGFR and inhibited self-renewal of the cells. Downregulation of DYRK1A also decreased tumor growth in nude mice after injection of GBM cells. Similarly, the authors found that pharmacological inhibition of DYRK $1 A$ reduced self-renewal of GBM cells and decreased tumor growth in nude mice injected with GBM cells.

The researchers then turned their attention to human glioma samples, finding that DYRK1A was more highly expressed in gliomas compared with normal brain tissue. They also identified a positive correlation between EGFR and DYRK1A expression in GBM samples.

Notably, the authors' data indicate that DYRK1A acts at the level of regulating EGFR stability rather than its kinase activity. They suggest that targeting DYRK1A, either alone or in combination with EGFR inhibitors, could have therapeutic benefit in EGFR-dependent GBMs. - MS

\section{NEURODEVELOPMENTAL DISORDERS}

\section{A therapy for Pretzel syndrome}

Treatment with the immunosuppressive drug rapamycin may have a therapeutic effect in patients with Pretzel syndrome, a rare neurodevelopmental disorder (Sci. Trans. Med. 5, 182ra53).

Pretzel syndrome is characterized by epilepsy, cognitive delay and a series of neuroanatomical abnormalities. The disease is caused by mutations in $\angle Y K 5$, which encodes an inhibitor of the mammalian target of rapamycin complex 1 (mTORC1). In mice, loss of Lyk5 affects neuronal migration and leads to the formation of clusters of misplaced neurons in the cortex similar to those observed in patients with Pretzel syndrome.

Whitney Parker et al. found that mTORC1 inhibition with rapamycin can prevent the

\title{
AUTISM SPECTRUM DISORDERS
}

\section{Mutual mutation mechanisms}

Researchers have identified altered endocannabinoid signaling as a common mechanism by which different mutations in the gene that encodes the synaptic protein neuroligin- 3 could cause autism (Neuron 78, 498-509).

Several mutations in the gene that encodes neuroligin-3 have been linked to autism, including a deletion mutation and a substitution at amino acid 451

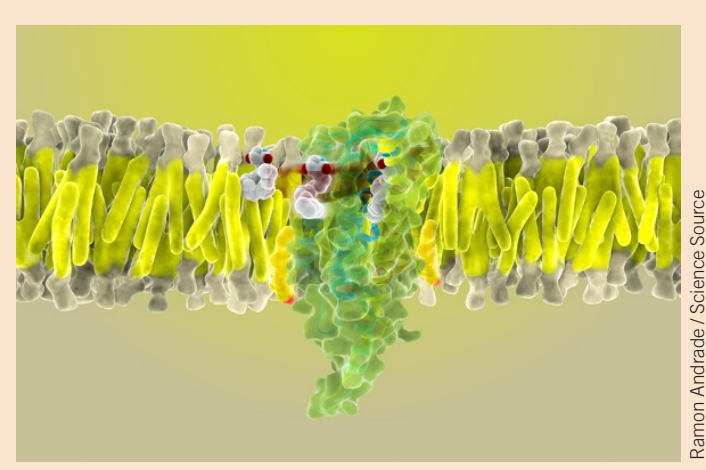
of the protein. Csaba Földy et al. examined the electrophysiological properties of synapses from inhibitory interneurons onto pyramidal neurons in the hippocampus with the goal of identifying synaptic dysfunction that would be commonly induced by both the mutations, possibly leading to autism. The researchers found that inhibitory neurotransmission from cholecystokinin-positive interneurons was enhanced in brain slices from mice with either mutation, whereas this was not the case with parvalbumin-positive interneurons.

Cholecystokinin interneurons express cannabinoid receptors, and deficits in cannabinoid signaling can enhance inhibitory neurotransmission. Földy et al. found that a cannabinoid receptor antagonist could enhance inhibitory neurotransmission in brain slices from wild-type mice, but could not do so in brain slices from either of the neuroligin-3-mutant mice, suggesting that they both had deficits in endocannabinoid signaling. How this mechanism could lead to the behavioral phenotypes associated with autism remains to be explored. $-E C$

cortical malformations caused by Lyk5 depletion in the mouse. As rapamycin also prevented a migration defect found in fibroblasts from patients with Pretzel syndrome, the authors treated five patients with rapamycin and reported that this resulted in a reduction in seizure frequency.

Although these findings need to be replicated in a larger cohort in a blinded trial, the results raise the possibility that $\mathrm{mTORC} 1$ inhibition may be useful for treating patients with this neurodevelopmental condition - JCL

\section{CARDIOVASCULAR DISEASES}

\section{Young blood helps the heart}

Enlargement of the heart-cardiac hypertrophy-occurs with aging and is thought to contribute to diastolic heart failure, a common form of heart failure in the elderly. Richard Lee and his colleagues now identify the cytokine GDF11 as a circulating antihypertrophic factor in mice whose levels decrease with age (Cell 153, 828-839).

In parabiosis experiments, in which the blood circulations of an old mouse and a young mouse are connected, the researchers found that cardiac hypertrophy in the old mouse was reversed after 4 weeks. The authors found a number of proteins whose abundance differed between blood plasma from young versus old mice. One of these was GDF11, which showed widespread tissue expression, with the highest levels in the spleen, and whose levels in both blood and spleen were higher in young mice as compared to old mice The researchers carried out functional studies to show that GDF11 inhibits cardiac hypertrophy of cultured cardiomyocytes and reverses cardiac hypertrophy in old mice. Raising the levels of GDF11 in the blood might therefore be beneficial for the aged heart. $-M B$

AGING

\section{A new target in progeria}

A recent study suggests that targeting the enzyme isoprenylcysteine carboxyl methyltransferase (ICMT) might have therapeutic benefit in a mouse model of a premature aging disease (Science http://dx.doi.org/10.1126/ science.1238880).

A number of progeroid disorders are caused by the accumulation of an aberrant form of prelamin $A$ at the nuclear envelope, leading to misshapen nuclei. Prelamin A is post translationally modified at its $C$ terminal by farnesylation and subsequent ICMT-mediated 